\title{
Year in Review 2018: Pediatric Mechanical Ventilation
}

\author{
Craig D Smallwood and Michael D Davis
}

\author{
Introduction \\ Pediatric ARDS \\ Factors Associated With a Risk of Mortality During Pediatric ARDS \\ Adjunct Therapy: INO \\ Sedation in the ICU and During Mechanical Ventilation \\ Delirium \\ Ventilator Management \\ Titrating PEEP \\ Neurally-Adjusted Ventilatory Assist After Pediatric Cardiac Surgery \\ Ventilation at Home \\ Quality and Safety \\ Ideal Body Weight \\ Ventilator-Associated Events and Pneumonias \\ Weaning and Liberation \\ Respiratory Therapist-Driven Weaning Protocol \\ Conclusions
}

\begin{abstract}
Mechanical ventilation is frequently used in pediatric patients to ensure adequate gas exchange, ameliorate respiratory distress, and enable resolution of pulmonary or other disorders. However, a number of important challenges remain in the pediatric population because there is a paucity of large-scale randomized controlled trials to generate data and inform clinical practice. This review summarizes a number of discoveries and advances that have been made in pediatric mechanical ventilation from June 2017 to December 2018. Key words: ICU; mechanical ventilation; pediatrics; pediatric ARDS; neurally-adjusted ventilatory assist. [Respir Care 2019;64(7):855-863. (0) 2019 Daedalus Enterprises]
\end{abstract}

\section{Introduction}

Mechanical ventilation is frequently used in pediatric patients to ensure adequate gas exchange, ameliorate re-

\footnotetext{
Dr Smallwood is affiliated with the Division of Critical Care Medicine, Department of Anesthesia, Critical Care and Pain Medicine, Boston Children's Hospital, Boston, Massachusetts. Dr Smallwood is affiliated with Harvard Medical School, Boston, Massachusetts. Dr Davis is affiliated with the Division of Pulmonary Medicine; Children's Hospital of Richmond at Virginia Commonwealth University, Richmond, Virginia.

Dr Smallwood discloses relationships with Ventec Life Systems and Mallinckrodt Pharmaceuticals. Dr Davis discloses a relationship with Mallinckrodt Pharmaceuticals.
}

spiratory distress, and enable resolution of pulmonary or other disorders. A number of important challenges remain in the pediatric population. For several practical reasons,

\footnotetext{
Dr Smallwood presented a version of this paper at the Year in Review of the AARC Congress 2018, held December 4-7, 2018, in Las Vegas, Nevada.

Correspondence: Craig D Smallwood PhD RRT, Division of Critical Care Medicine, Department of Anesthesia, Critical Care and Pain Medicine, Boston Children's Hospital, 300 Longwood Avenue, Bader Building 634, Boston, MA 02115. E-mail: craig.smallwood@childrens.harvard.edu.
}

DOI: 10.4187/respcare.07029 


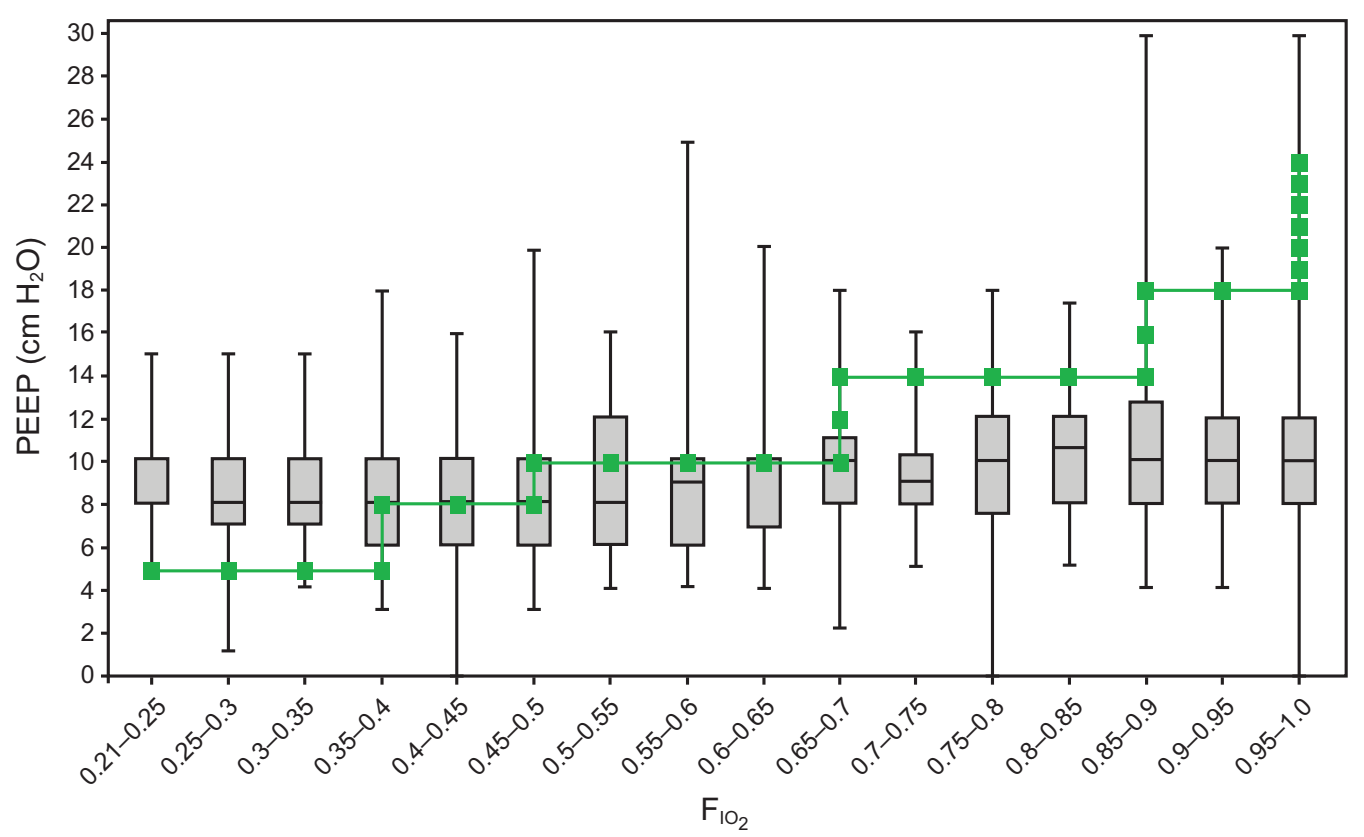

Fig. 1. All pediatric ARDS PEEP- $\mathrm{F}_{\mathrm{IO}_{2}}$ combinations. Actual PEEP values as a function of actual $\mathrm{F}_{\mathrm{IO}_{2}}$ levels (median [bar] and interquartile range [box]) for all the subjects with pediatric ARDS for the first day of mechanical ventilation after a pediatric ARDS diagnosis. The superimposed line represents the ARDS Network protocol target combinations of PEEP- $\mathrm{F}_{1 \mathrm{I}_{2}}$. In general, the clinicians used more PEEP than recommended when $\mathrm{F}_{\mathrm{IO}_{2}}$ was $<0.4$ and used less PEEP than recommended when $\mathrm{F}_{\mathrm{IO}_{2}}$ was $>0.5$. Median $\mathrm{PEEP}$ did not exceed $10 \mathrm{~cm} \mathrm{H}_{2} \mathrm{O}$, regardless of $\mathrm{F}_{\mathrm{IO}_{2}}$. From Reference 1, with permission.

there has been a paucity of large-scale randomized controlled trials to generate data to inform clinical practice during pediatric mechanical ventilation. In many cases, it is necessary to extrapolate from studies conducted in adult populations, but many differences exist between mechanically ventilated adults and children. This review summarizes a number of discoveries and advances within the field of pediatric mechanical ventilation that were published from June 2017 to December 2018.

\section{Pediatric ARDS}

There are a number of similarities in the pathophysiology of ARDS in adults and children; both manifest with reductions in respiratory system compliance and the need for mechanical ventilation, and carry a high incidence of mortality. However, pediatric-specific practice patterns, comorbidities, and differences in outcome necessitate a pediatric-specific definition and even differences in treatment strategies. Pediatric ARDS is characterized by acute onset, acute pulmonary parenchymal disease on the chest radiograph, and an oxygenation index $\geq 4$ (oxygenation index $\left.=\left(\left[\overline{\mathrm{P}}_{\mathrm{aw}} \times \mathrm{F}_{\mathrm{IO}_{2}}\right) / \mathrm{P}_{\mathrm{aO}_{2}}\right]\right)$ or an oxygen saturation index of $\geq 5$ (oxygen saturation index $=\left[\overline{\mathrm{P}}_{\mathrm{aw}} \times \mathrm{F}_{\mathrm{IO}_{2}}\right) / \mathrm{S}_{\mathrm{pO}_{2}}$, provided $\mathrm{S}_{\mathrm{pO}_{2}}$ is $\leq 97 \%$ ]). Pediatric ARDS is stratified as mild, moderate, or severe. Patients with severe pediatric ARDS have the highest likelihood of mortality. Overall, the mortality rate for children with pediatric ARDS is $\sim 18 \%$.

\section{Factors Associated With a Risk of Mortality During Pediatric ARDS}

Khemani et $\mathrm{al}^{1}$ sought to evaluate the relationship between PEEP and mortality during pediatric ARDS. It was a multicenter retrospective study that combined data of 1,134 mechanically ventilated children with pediatric ARDS from 4 previous investigations. The overall mortality rate observed in the study was $18.6 \% .^{1}$ Because it is apparent that the application of high levels of inspired oxygen and PEEP are associated with pediatric ARDS severity, the investigators cleverly calculated the difference between the applied PEEP (by using a median value in the first day of pediatric ARDS) and the recommended PEEP from the lower arm of the ARDS Network (ARDSnet) PEEP- $\mathrm{F}_{\mathrm{IO}_{2}}$ grid. ${ }^{1,2}$ Interestingly, the investigators described an overwhelming propensity to apply a PEEP of $6-10 \mathrm{~cm} \mathrm{H}_{2} \mathrm{O}$ and very infrequently to apply levels $>10 \mathrm{~cm} \mathrm{H}_{2} \mathrm{O}$ (Fig. 1). The investigators note the main finding of the study, that, because the difference between applied and ARDSnet recommended PEEP increases, so too does the likelihood of mortality. ${ }^{1}$ After adjusting for a variety of clinical factors, including the level of hypoxemia, ventilator settings, severity of illness, use of inotropes, comorbidities, PEEP lower than recommended was independently associated with higher mortality (odds ratio $2.05,95 \%$ CI 1.32-3.17). ${ }^{1}$

In addition, the investigators provide survival curve analyses for the entire cohort and subgrouped subjects based 
on $\mathrm{P}_{\mathrm{aO}_{2}} / \mathrm{F}_{\mathrm{IO}_{2}}$ ranges. ${ }^{1}$ Overall, a PEEP lower than recommended by protocol for a given $\mathrm{F}_{\mathrm{IO}_{2}}$ was associated with higher mortality $(P<.001)$. However, when subgrouping by the initial $\mathrm{P}_{\mathrm{aO}} / \mathrm{F}_{\mathrm{IO}_{2}}$, the only statistically significant finding was for the range between 100 and $200(P=.004) .{ }^{1}$ This finding indicates that patients in the initial $\mathrm{P}_{\mathrm{aO}} / \mathrm{F}_{\mathrm{IO}_{2}}$ range between 100-200 may see the greatest benefit from modifying application of PEEP and that future studies should focus not only on evaluating the general concepts prospectively but perhaps especially in this subgroup.

When it comes to assessing and accounting for variance in ventilator management, the study was observational, and, therefore, the study design did not allow for the protocolization of clinical care. ${ }^{1}$ Important questions, therefore, should be raised regarding the adequacy of controlling for confounding factors, such as gas exchange targets, tidal volume, and pressure targets; use of sedatives and paralytic agents; diagnosis; and application and timing of adjunct therapies, such as inhaled nitric oxide (INO) and other institutional factors. The investigators applied a formal statistical test to account for interaction between hospitals, but this finding was negative $(P=.4){ }^{1}$

However, the investigators further explored this question by deriving the mortality risk model separately for the 2 principle institutions that contributed data (Children's Hospital of Los Angeles and Children's Hospital of Philadelphia). After controlling for potential confounders, the mortality risk associated with the difference in PEEP and ARDSnet recommended remained only for the Children's Hospital of Los Angeles dataset (odds ratio 2.09, 95\% CI 1.26-3.46); the Children's Hospital of Philadelphia dataset demonstrated a reduction in mortality risk (odds ratio 0.87 , 95\% CI 0.32 2.35 ), although the $\mathrm{CI}$ is wide, so it is difficult to draw specific conclusions. ${ }^{1}$ Overall, the study by Khemani et $\mathrm{al}^{1}$ is an important contribution to the pediatric ARDS literature and highlights interesting practice patterns that are associated with an increased risk of mortality. Although these finding should certainly inform future clinical trials, it is difficult to assign a causal relationship between the PEEP difference and mortality and, therefore, extrapolating these finding to modify current clinical practice would not be prudent.

\section{Adjunct Therapy: INO}

The physiologic rationale for applying INO to children with pediatric ARDS derives from the fact that INO is a pulmonary vasodilator and modulator of endothelial function. Conceivably, dilating the pulmonary vasculature could improve ventilation-perfusion matching, which results in improved oxygenation and the ability to wean the patient off mechanical ventilator. However, the oxygenation effects have been noted to be only transient, and multiple studies in both adults and children demonstrated no mortality benefit with the application of INO during ARDS..$^{3-5}$ However, INO is still applied to improve hypoxemia in up to $13 \%$ of children with pediatric ARDS. ${ }^{6}$ On one hand, it would be reasonable for a clinical scientist to question why more studies that evaluate the mortality benefit of INO in pediatric ARDS is necessary. On the other hand, a practical bedside clinician may argue that a trial of INO is generally benign, easy to conduct, and may be used as a bridge to other supportive therapies, such as high-frequency oscillatory ventilation or initiation of extracorporeal membrane oxygenation. Indeed, the application of INO in the pediatric ICU is something that many clinicians wrestle with.

To that end, Bhalla et $\mathrm{al}^{7}$ published a multi-center retrospective cohort study of 499 mechanically ventilated children with pediatric ARDS, and conducted a subgroup analysis of 143 subjects who received INO. They applied propensity score matching, a statistical method that attempts to estimate the effect of a treatment by accounting for covariates that are associated with receiving the treatment in the first place. ${ }^{7}$ These covariates included age, weight, pediatric ARDS trigger and/or etiology, comorbidities, initial oxygenation index, vasoactive support, pediatric risk of mortality (PRISM III) score, acute neurologic injury, cardiac arrest, and admitting hospital. On comparison between a cohort that received INO and a cohort that did not, the primary diagnosis of pneumonia, higher initial oxygenation index, and more-vasoactive support were associated with initiation of INO. ${ }^{7}$ In the unadjusted analysis, mortality was noted to be higher in the INO treatment group (25.2 vs $16.3 \%, P=.02)$ and also had worsening 28 -d ventilator-free days (10 vs $17 \mathrm{~d}$, $P<.001)^{7}$

However, because these data were unadjusted and INO is frequently only initiated in children with a more severe degree of hypoxemia, the investigators applied the propensity score matched analysis. Importantly, in this matched cohort, INO was not associated with mortality (odd ratio $1.3,95 \%$ CI $0.56-3.0$ ) or 28 -d ventilator-free days (incidence rate ratio $0.91,95 \%$ CI $0.80-$ 1.04). ${ }^{7}$ Overall, there was no benefit to mortality or 28-d ventilator-free days in those subjects who received INO. However, what about those subjects who demonstrated a positive oxygenation response to INO? In a secondary analysis, mortality was not associated with stratified oxygenation response $(P=.52)$; however, in the case of children who did not demonstrate a response to INO, the investigators noted an increased risk of having zero ventilator-free days (odds ratio 6.1, 95\% CI $1.4-26.3 ; P=.02) .{ }^{7}$ This finding is of scientific interest but little clinical benefit. Although it is interesting to identify a subgroup of patients who have a difference in outcome based on clinical response to a 
therapy, applying INO just to find out how many ventilator-free days is not practical or wise.

One of the most important limitations of the present study was the fact that the investigators were only able to match $61.5 \%$ of the children who received INO with those who did not receive INO. ${ }^{7}$ Therefore, the study was powered only to identify fairly large differences in mortality and ventilator-free days. With a larger cohort, it is possible that a smaller effect size could be identified. Overall, INO in the study by Bhalla et $\mathrm{al}^{7}$ demonstrates that INO treatment in pediatric ARDS is not associated with a significant improvement in mortality or ventilator-free days. This is one of the first studies to demonstrate a potential harm for applying INO to children with pediatric ARDS; however, this conclusion is based on the unadjusted analysis, so the clinical utility is unclear.

\section{Sedation in the ICU and During Mechanical Ventilation}

\section{Delirium}

Delirium is characterized by an acute change in mental status with confusion or an unexplained altered level of consciousness that occurs frequently in pediatric patients who are critically ill, including those who are on mechanical ventilation. ${ }^{8}$ Delirium can be a symptom of critical illness or an adverse effect of interventions used during the administration of care, for example, sedation. ${ }^{8}$ The relationships between delirium and mechanical ventilation are relevant to clinicians, and methods for reducing both of these is desired.

Traube et $\mathrm{al}^{8}$ conducted a prospective longitudinal cohort study that evaluated delirium in pediatric subjects who were critically ill. They found that both benzodiazepine administration and mechanical ventilation were independent predictors of delirium in the subjects who were critically ill. They also found that delirium is associated with the requirement and duration of mechanical ventilation and mortality in this patient population, which suggests that minimizing the need for benzodiazepine use could reduce delirium, mechanical ventilation duration, and mortality in pediatric patients who are critically ill, but future prospective trials are needed.

\section{Ventilator Management}

\section{Titrating PEEP}

In children with hypoxic respiratory failure and on mechanical ventilation, PEEP is titrated to improve oxygenation through the recruitment and stabilization of alveoli. However, PEEP can both exacerbate or reduce the degree of lung injury. ${ }^{9}$ Unfortunately, little guidance exists in the pediatric literature to inform the clinician at the bedside adjusting PEEP. In most cases, the rationale for increasing PEEP is to improve oxygenation and reduce lung injury by improving end-expiratory lung volume and compliance, by reducing the dead-space and shunt fractions, and the overall degree of ventilation-perfusion mismatch. ${ }^{10-12} \mathrm{In}-$ deed, the use of moderate and even high levels of PEEP have been shown to be safe in the pediatric population. ${ }^{13-16}$ However, widespread and consistent application has not been recommended. ${ }^{17,18}$ Decisions are inherently heterogeneous and vary by institution and medical unit, and among individual clinicians. ${ }^{19}$

Our group sought to quantify the amount of time required for oxygenation and pulmonary compliance to equilibrate after an increase or decrease in PEEP. ${ }^{20}$ The study was retrospective, and a total of 200 subjects were enrolled and included 1,150 PEEP changes; however, only 54 of these subjects and 171 PEEP changes were included in the analysis based on quality and data completion standards. ${ }^{20}$ For each PEEP change, data were collected for $90 \mathrm{~min}$ after the increase. ${ }^{20}$ The time to $90 \%$ of the overall improvement in oxygenation and pulmonary compliance was computed; this was done to normalize the improvement and compare all results together. A PEEP change case was classified as either a responder (improvement in oxygenation) or non-responder (either no improvement or worsening in oxygenation).

In responders, the time to $90 \%$ required $38 \mathrm{~min}$ for compliance and $71 \mathrm{~min}$ for oxygenation (Fig. 2). ${ }^{20}$ The fact that compliance preceded changes in oxygenation in the setting of hypoxic respiratory failure makes sense. After an improvement in compliance, additional time was required to carry oxygen to the alveolar-capillary membrane. ${ }^{20}$ Redistribution of blood flow within the pulmonary system may account for the delayed improvement in oxygenation. In addition, we observed that the time to achieve peak oxygenation after an increase in PEEP was dependent on the severity of hypoxic respiratory failure; lung dysfunction was associated with a longer equilibration time $(P=.001) .{ }^{20}$ In non-responders, both compliance and oxygenation deteriorated at essentially the same time (65 min for oxygenation and $64 \mathrm{~min}$ for compliance). ${ }^{20}$

Limitations to the study include the following: it was conducted at a single center, it was retrospective, the reason for increasing PEEP was not controlled, and the degree to which PEEP was titrated was only $1-3 \mathrm{~cm} \mathrm{H}_{2} \mathrm{O} .{ }^{20}$ Cardiac output was also not measured and could have been a confounding factor. However, heart rate, mean arterial blood pressure, $\mathrm{CO}_{2}$ elimination, and breathing frequency were recorded throughout the 90-min period and reported no changes except for heart rate, which decreased on average from 120 to 118 beats/min $(P=.02) .{ }^{20}$ If cardiac output had accounted for changes in oxygenation, then 

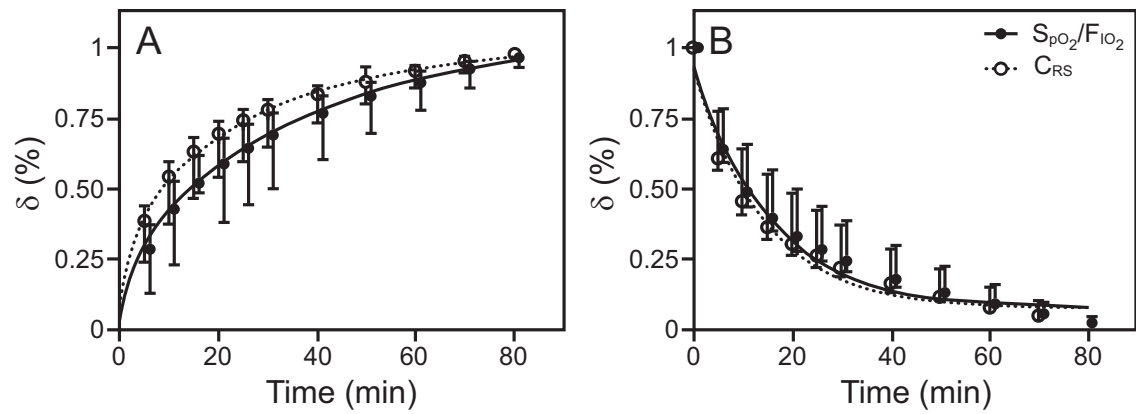

Fig. 2. Comparison of increases in dynamic pulmonary compliance and oxygenation. Mean $\pm \mathrm{SD}$ values are denoted by the circles and whiskers; $\delta(\%)$ is the change in either oxygen saturation index (OSI) (OSI $\left.=\mathrm{F}_{1 \mathrm{O}_{2}} \times \overline{\mathrm{P}}_{\mathrm{aw}} \times 100 / \mathrm{S}_{\mathrm{pO}_{2}}\right)$ or respiratory system compliance $\left(\mathrm{C}_{\mathrm{RS}}\right)$ relative to the maxima at each time point. A: Responders (those who had an increase in oxygenation after PEEP increase), OSI improved by $13 \%$ and $\mathrm{C}_{\mathrm{RS}}$ improved by $14 \%$ during the monitoring time frame in responders. B: Non-responders (no change in oxygenation or worsening after PEEP increase). From Reference 20, with permission.

increases would be anticipated in blood pressure and heart rate. Because this was not the case in the present study, it is unlikely this played an important role. ${ }^{20}$ It is tempting to suggest that these finding indicate a "wait and see" approach to PEEP management, but further prospective evaluation of these findings are indicated. If validated, these findings may aid bedside clinicians in determining the optimum time required between PEEP changes.

\section{Neurally-Adjusted Ventilatory Assist After Pediatric Cardiac Surgery}

Children on mechanical ventilation after cardiac surgery require special care and a mechanical ventilation strategy that often differs markedly from lung-protective ventilation. Intrathoracic pressures can reduce venous return and increase right ventricular afterload, and, as such, a "higher tidal volume, lower breathing frequency" strategy is implemented to reduce the mean airway pressure while still maintaining an adequate minute ventilation. ${ }^{21,22}$ Neurallyadjusted ventilatory assist (NAVA) is a mode of ventilation that uses the electrical activity of the diaphragm, measured by a catheter placed in the esophagus, to trigger the mechanical ventilator and deliver positive pressure based on the detected degree of electrical activity. ${ }^{23}$ By virtue of its triggering mechanism, which is independent of the breathing circuit, NAVA could have potential benefits for those patients with hyperdynamic cardiac activity or those supported with a ventricular assist device. Because the heart sits so close to the trachea, the hyperdynamic cardiac activity can cause flow oscillations in the breathing circuit and lead to auto-triggering with tradition flow and pressure trigger.

Crulli et $\mathrm{a}^{24}$ reported clinical experience with NAVA applied to children after cardiac surgery. The study was retrospective and included vital sign data, mechanical ventilation parameters, gas exchange, and arterial blood gas data. A total of 28 subjects were enrolled in the study, including those who were receiving noninvasive or invasive NAVA. ${ }^{24}$ Of interest, the investigators report a reduction in peak inspiratory pressure from $20.9 \mathrm{~cm} \mathrm{H}_{2} \mathrm{O}$ during pressure- or volume-cycled ventilation to $15.1 \mathrm{~cm} \mathrm{H}_{2} \mathrm{O}$ during NAVA $(P<.001){ }^{24}$ The investigators did not report mean airway pressure, but it may be assumed that it decreased because breathing frequency was similar before and during NAVA. It is noted that the tidal volume was $0.5 \mathrm{~mL} / \mathrm{kg}$ lower during NAVA compared with baseline, but this finding was not statistically significant (Table 1). No other factors were noted to be different in the study before versus during NAVA. ${ }^{24}$ It is difficult to make any clinical recommendations based on these results because the study design precluded one's ability to assess pulmonary blood flow or surrogates thereof. The investigators note that overall, NAVA was tolerated well and a number of scenarios can be imagined in which NAVA may prove to be beneficial; however, as the investigators conclude, prospective trials are needed to assess the effect of NAVA on short-term functional outcomes, such as pulmonary vascular resistance and blood flow, but also on clinical outcomes, including duration of ventilation, length of ICU stay, and mortality. ${ }^{24}$

\section{Ventilation at Home}

Improvements in technology and changes in mechanical ventilation strategies have led to an increase in long-term ventilation outside of ICUs. Mechanical ventilation at a long-term facility or at home reduces the census burden on ICUs and financial cost of long ICU stays. It also allows patients who require mechanical ventilation to live in the comfort of their homes and may reduce the overall hospital burden in some cases. Amirnovin et $\mathrm{al}^{25}$ conducted a retrospective analysis over a 26-y period that evaluated the trends of pediatric subjects who required home ventilation after hospital- 
Table 1. Vital Sign and Laboratory Values Before and After Invasive NAVA

\begin{tabular}{|c|c|c|c|c|c|}
\hline \multirow[t]{2}{*}{ Parameter } & \multicolumn{2}{|c|}{$\begin{array}{l}\text { 4-h Period Before } \\
\text { NAVA }\end{array}$} & \multicolumn{2}{|c|}{$\begin{array}{l}\text { 4-h Period With } \\
\text { NAVA }\end{array}$} & \multirow[t]{2}{*}{$P$} \\
\hline & $n$ & Results & $n$ & Results & \\
\hline \multicolumn{6}{|l|}{ Vital signs, mean $\pm \mathrm{SD}$} \\
\hline Frequency, breaths/min & 27 & $31 \pm 8$ & 27 & $31 \pm 9$ & .81 \\
\hline Heart rate, beats/min & 27 & $128 \pm 24$ & 27 & $128 \pm 23$ & .98 \\
\hline Mean arterial blood pressure, $\mathrm{mm} \mathrm{Hg}$ & 19 & $63 \pm 10$ & 19 & $61 \pm 9$ & .17 \\
\hline \multicolumn{6}{|l|}{ Oxygenation } \\
\hline $\mathrm{F}_{\mathrm{IO}_{2}}$, mean $\pm \mathrm{SD} \%$ & 27 & $52 \pm 22$ & 27 & $52 \pm 23$ & .75 \\
\hline $\mathrm{S}_{\mathrm{pO}_{2}}$, mean $\pm \mathrm{SD} \%$ & 27 & $96 \pm 7$ & 27 & $96 \pm 7$ & .49 \\
\hline $\mathrm{S}_{\mathrm{pO}_{2}} / \mathrm{F}_{\mathrm{IO}_{2}}$, median (IQR) & 27 & $194(157-275)$ & 27 & $198(165-287)$ & .09 \\
\hline \multicolumn{6}{|l|}{ Ventilation } \\
\hline Tidal volume, mean $\pm \mathrm{SD} \mathrm{mL} / \mathrm{kg}$ & 27 & $6.8 \pm 2.3$ & 27 & $6.3 \pm 3.1$ & .24 \\
\hline PEEP, median (IQR) $\mathrm{cm} \mathrm{H}_{2} \mathrm{O}$ & 27 & $5(5-7)$ & 27 & $5(5-7)$ & .41 \\
\hline \multicolumn{6}{|c|}{$\begin{array}{l}\text { Laboratory values for subjects with data available in pre- and } \\
\text { post-NAVA periods }\end{array}$} \\
\hline $\mathrm{pH}$, mean $\pm \mathrm{SD}$ & 9 & $7.35 \pm 0.06$ & 9 & $7.36 \pm 0.04$ & .51 \\
\hline $\mathrm{P}_{\mathrm{aCO}_{2}}$, mean $\pm \mathrm{SD} \mathrm{mm} \mathrm{Hg}$ & 9 & $47.6 \pm 8.6$ & 9 & $45.2 \pm 7.8$ & .20 \\
\hline $\mathrm{P}_{\mathrm{aO}_{2}}$, mean $\pm \mathrm{SD} \mathrm{mm} \mathrm{Hg}$ & 8 & $158 \pm 82$ & 8 & $148 \pm 77$ & .67 \\
\hline $\mathrm{P}_{\mathrm{aO}_{2}} / \mathrm{F}_{\mathrm{IO}_{2}}$, median $(\mathrm{IQR}) \mathrm{mm} \mathrm{Hg}$ & 8 & $271(137-419)$ & 8 & $304(97-432)$ & .67 \\
\hline Lactates, mean $\pm \mathrm{SD} \mathrm{mmol} / \mathrm{L}$ & 7 & $1.15 \pm 0.54$ & 7 & $1.22 \pm 0.4$ & .61 \\
\hline \multicolumn{6}{|l|}{ Laboratory values, including all data } \\
\hline $\mathrm{pH}$, mean $\pm \mathrm{SD}$ & 10 & $7.36 \pm 0.06$ & 24 & $7.39 \pm 0.04$ & NA \\
\hline $\mathrm{P}_{\mathrm{aCO}_{2}}$, mean $\pm \mathrm{SD} \mathrm{mm} \mathrm{Hg}$ & 10 & $48.0 \pm 8.2$ & 24 & $45.1 \pm 6.2$ & NA \\
\hline $\mathrm{P}_{\mathrm{aO}_{2}}$, mean $\pm \mathrm{SD} \mathrm{mm} \mathrm{Hg}$ & 8 & $158 \pm 83$ & 18 & $123 \pm 63$ & NA \\
\hline $\mathrm{P}_{\mathrm{aO}_{2}} / \mathrm{F}_{\mathrm{IO}_{2}}$, median $(\mathrm{IQR}) \mathrm{mm} \mathrm{Hg}$ & 8 & $272(137-419)$ & 18 & $206(146-365)$ & NA \\
\hline Lactates, mean $\pm \mathrm{SD} \mathrm{mmol} / \mathrm{L}$ & 10 & $1.15 \pm 0.46$ & 21 & $1.26 \pm 0.45$ & NA \\
\hline \multicolumn{6}{|l|}{$\begin{array}{l}\text { From Reference } 24 . \\
\text { NAVA = neurally-adjusted ventilatory assist } \\
\text { IQR = interquartile range } \\
\mathrm{NA}=\text { not applicable }\end{array}$} \\
\hline
\end{tabular}

ization. They found an increase in the frequency of the subjects being discharged with home ventilation over that time period; they also reported an increase in subject complexity in more recent years, which they suggest could be due to more advanced ventilator technology, particularly the advent of internal PEEP valves in home ventilators. ${ }^{25}$ Although this report was based solely on a retrospective chart review, it does suggest an increase in the prevalence of home ventilation, perhaps due to changes in technology and standards of ventilation that require ICU admission. As more remote and automatic monitoring and telemedicine enter the standard of care, it is likely that home ventilation of complex pediatric patients will continue to increase.

\section{Quality and Safety}

\section{Ideal Body Weight}

The utility of ideal body weight (IBW) as a method of normalizing tidal volumes during mechanical ventilation has been well established in neonates and in pediatric and adult patients. Indeed, IBW is simply a rough surrogate for total lung volume because patients who are taller or who are male tend to have a larger lung capacity. Despite this, a universal method for calculating IBW has not been established in the pediatric population.

Bilharz et $\mathrm{al}^{26}$ studied 58 pediatric subjects who received mechanical ventilation and compared 3 methods of calculating IBW with actual body weight. They used BlandAltman analysis to quantify the individual agreement between each method and the actual body weight (Fig. 3). ${ }^{26}$ They also reported the number of subjects who would have a "clinically important error," which was defined as a difference of $\geq 10 \%$ between actual body weight and IBW. The Bland-Altman analysis showed that the difference between the actual body weight and IBW increased in the subjects who were larger and was most apparent in those $>25 \mathrm{~kg} .{ }^{26}$ In addition, the majority of the subjects (51.7-56.9\%) demonstrated a clinically important error. ${ }^{26}$ Analysis of these data underlines the importance of obtaining height measurements and computing IBW to facil- 


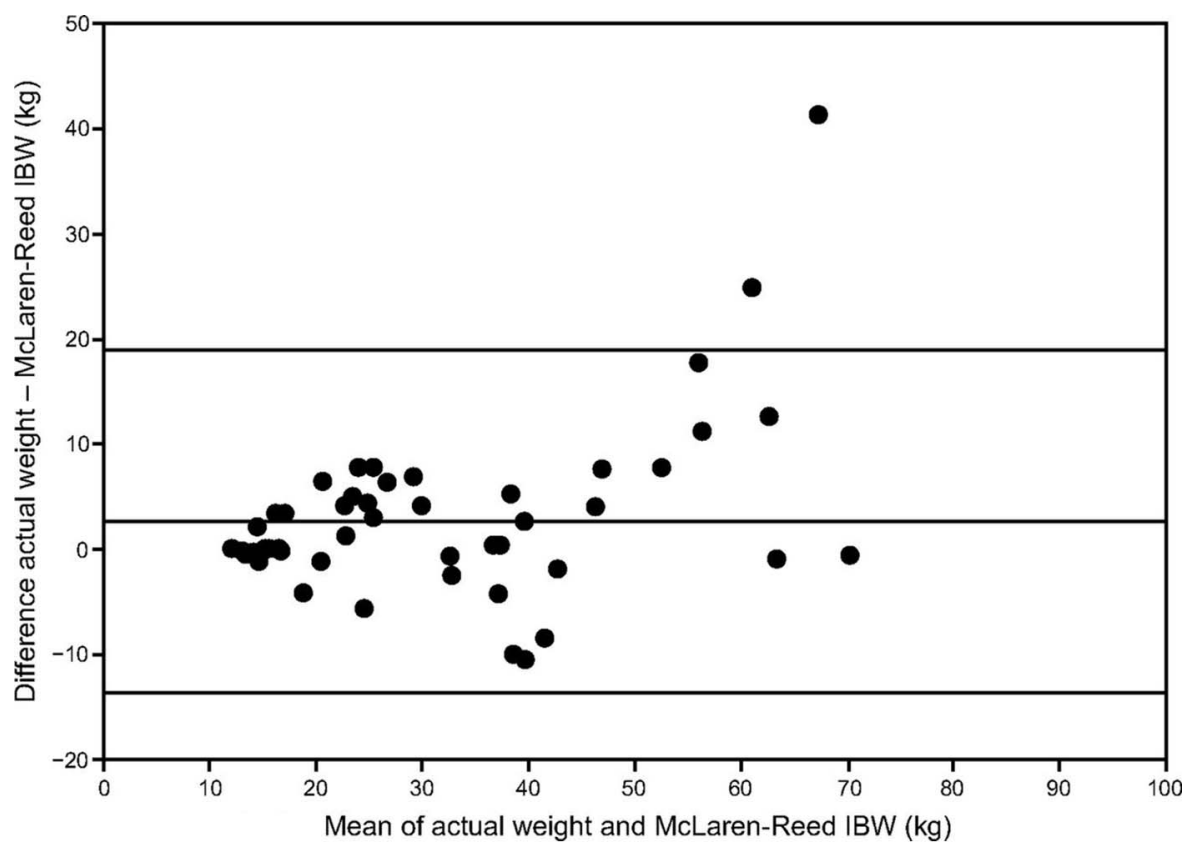

Fig. 3. Bland-Altman plot for the McLaren-Read ideal body weight (IBW) method in male and female subjects ages 2-20 y. The center line denotes bias; outside lines show upper and lower limits. From Reference 26.

itate accurate tidal volume measurements in the pediatric ICU. The investigators also noted that a method that uses body mass index data is indicated for patients $>2 \mathrm{y}$ of age. ${ }^{26}$

In a large, multi-center post hoc analysis, Ward et $\mathrm{al}^{27}$ examined the variability in the pediatric IBW calculation and examined 4 methods. The investigators sought to identify the "best" method for IBW calculation, defined as that that (1) could be calculated in the majority of subjects, (2) demonstrated good agreement with other methods, and (3) would conceivably lead to the use of a lower tidal volume. ${ }^{27}$ It was noted that the other methods for calculating IBW had a large variance, especially in those subjects $>10 \mathrm{y}$ of age. The investigators recommend that the McLaren method be used because it was able to be applied in $>90 \%$ of the population and demonstrated good agreement with the other methods. ${ }^{27}$

\section{Ventilator-Associated Events and Pneumonias}

In 2013, the Centers for Disease Control and Prevention instituted a national surveillance definition that replaced ventilator-associated pneumonia (VAP) with a ventilatorassociated event (VAE) and specifically outlined a pediatric definition of VAE. Pediatric VAEs are identified by a deterioration in respiratory status after a period of stability or improvement during mechanical ventilation. The stable period of ventilation is defined as $\geq 2$ calendar days in which the daily minimum mean airway pressure and $\mathrm{F}_{\mathrm{IO}_{2}}$ are unchanged or decreasing. If, after these 2 stable days are followed by an increase in mean airway pressure $\geq 4 \mathrm{~cm} \mathrm{H}_{2} \mathrm{O}$ or $\mathrm{F}_{\mathrm{IO}_{2}} \geq 25 \%$ for $2 \mathrm{~d}$, then a VAE has occurred. The overall incidence of VAE in children is $\sim 3$ per 1,000 ventilator days and is associated with a substantial risk of mortality and morbidity. ${ }^{29}$

Chomton et $\mathrm{al}^{30}$ conducted a single-center retrospective investigation of 284 children on mechanical ventilation. In this cohort, $10.6 \%$ of the subjects met Centers for Disease Control and Prevention criteria for VAP, 7 per 1,000 ventilator days. ${ }^{30}$ VAP was associated with a longer duration of ventilation and ICU length of stay. Use of bronchoscopy $(P=.03)$, continuous enteral feeding $(P=.02)$, and pediatric ARDS prevalence $(P=.03)$ were higher in children with VAP than those without VAP. ${ }^{30}$ Manipulations of the airway during bronchoscopy and enteral feeding may be associated with VAP because they both provide a mechanism for contamination of the breathing circuit or aspiration of gastric contents. However, because neither of these were directly measured in the study, it is difficult to assign causation. Unfortunately, the investigators did not report the number of subjects that was classified as VAE from within the larger cohort but do note that, of the 30 subjects who had a VAP, 17 also met criteria for adult VAE (different PEEP and $\mathrm{F}_{\mathrm{IO}_{2}}$ thresholds). ${ }^{30}$

Pediatric VAE is an important screening tool in mechanically ventilated triggers. It facilitates the objective identification of events that are associated with an increased pediatric ICU length of stay, use of mechanical ventilation, and mortality. The next step will be instituting prac- 
tices aimed at reducing the probability of a VAE in the pediatric ICU and striving to drive that rate to zero.

\section{Weaning and Liberation}

\section{Respiratory Therapist-Driven Weaning Protocol}

Since the development of standardized spontaneous breath trials and demonstration of their value in weaning patients from mechanical ventilation, development of protocols to facilitate conduction of these trials have been proposed at many centers in numerous formats. ${ }^{31,32}$ Success of these protocols are evaluated primarily on improved patient outcomes. However, a lack of improved outcomes may be indicative of poor adherence to the protocol rather than of an ineffective protocol.

Krawiec et al $^{33}$ initiated a respiratory therapist-driven protocol designed to increase early evaluation of patient readiness for spontaneous breath trials in pediatric subjects on mechanical ventilation by enabling respiratory therapists to initiate spontaneous breath trials if the subjects met criteria that indicated cardiopulmonary stability and a decrease in sedation. They then conducted a retrospective chart review to evaluate the effect of this protocol on patient outcomes, including duration of mechanical ventilation, length of ICU stay, and length of hospital stay. They also reported institutional adherence to the protocol. No difference in subject outcomes was found after initiation of the respiratory therapist-driven protocol; however, they reported a $56 \%$ institutional adherence to the new protocol, which may indicate that the protocol was not effectively executed rather than ineffective at altering patient outcomes. ${ }^{33}$ Of note, they did report an increase in noninvasive ventilation usage after initiation of the protocol, which may have been due to an increased respiratory therapist role or presence during patient weaning. ${ }^{33}$

\section{Conclusions}

From June 2017 to December 2018, peer-reviewed reports related to pediatric mechanical ventilation were published that evaluated the management and mortality of pediatric ARDS, the use of INO, the relevance of delirium, titration of PEEP, use of NAVA, advances in home ventilation, calculation of IBW, screening of pediatric VAEs, and effectiveness of respiratory therapist-driven weaning protocols. The art and science of pediatric mechanical ventilation is by no means settled, and incremental improvements in our understanding continue to be made. It is essential for respiratory clinicians to inform their clinical practice by assessing the available literature and to plan future studies based on the greatest need in the field.

\section{REFERENCES}

1. Khemani RG, Parvathaneni K, Yehya N, Bhalla AK, Thomas NJ, Newth CJL. Positive end-expiratory pressure lower than the ARDS Network protocol is associated with higher pediatric acute respiratory distress syndrome mortality. Am J Respir Crit Care Med 2018; 198(1):77-89.

2. Acute Respiratory Distress Syndrome Network, Brower RG, Matthay MA, Morris A, Schoenfeld D, Thompson BT, Wheeler A. Ventilation with lower tidal volumes as compared with traditional tidal volumes for acute lung injury and the acute respiratory distress syndrome. N Engl J Med 2000;342(18):1301-1308.

3. Adhikari NK, Burns KE, Friedrich JO, Granton JT, Cook DJ, Meade MO. Effect of nitric oxide on oxygenation and mortality in acute lung injury: systematic review and meta-analysis. BMJ 2007; 334(7597):779.

4. Afshari A, Brok J, MøllerAM, Wetterslev J. Inhaled nitric oxide for acute respiratory distress syndrome and acute lung injury in adults and children: a systematic review with meta-analysis and trial sequential analysis. Anesth Analg 2011;112(6):1411-1421.

5. Afshari A, Brok J, Moller AM, Wetterslev J. Inhaled nitric oxide for acute respiratory distress syndrome (ARDS) and acute lung injury in children and adults. Cochrane Database Syst Rev 2010;(7):CD002787.

6. Munshi L, Rubenfeld G, Wunsch H. Adjuvants to mechanical ventilation for acute respiratory distress syndrome. Intensive Care Med 2016;42(5):775-778.

7. Bhalla AK, Yehya N, Mack WJ, Wilson ML, Khemani RG, Newth CJL. The association between inhaled nitric oxide treatment and ICU mortality and 28-day ventilator-free days in pediatric acute respiratory distress syndrome. Crit Care Med 2018;46(11):1803-1810.

8. Traube C, Silver G, Gerber LM, Kaur S, Mauer EA, Kerson A, et al. Delirium and mortality in critically ill children: epidemiology and outcomes of pediatric delirium. Crit Care Med 2017;45(5):891-898.

9. Kallet RH. Should PEEP titration be based on chest mechanics in patients with ARDS? Respir Care 2016;61(6):876-890.

10. von Ungern-Sternberg BS, Regli A, Schibler A, Hammer J, Frei FJ, Erb TO. The impact of positive end-expiratory pressure on functional residual capacity and ventilation homogeneity impairment in anesthetized children exposed to high levels of inspired oxygen. Anesth Analg 2007;104(6):1364-1368, table of contents.

11. Rimensberger PC, Pache JC, McKerlie C, Frndova H, Cox PN. Lung recruitment and lung volume maintenance: a strategy for improving oxygenation and preventing lung injury during both conventional mechanical ventilation and high-frequency oscillation. Intensive Care Med 2000;26(6):745-755.

12. Papadakos PJ, Lachmann B. The open lung concept of mechanical ventilation: the role of recruitment and stabilization. Crit Care Clin 2007;23(2):241-250, ix-X.

13. Duff JP, Rosychuk RJ, Joffe AR. The safety and efficacy of sustained inflations as a lung recruitment maneuver in pediatric intensive care unit patients. Intensive Care Med 2007;33(10):1778-1786.

14. Kheir JN, Walsh BK, Smallwood CD, Rettig JS, Thompson JE, Gómez-Laberge C, et al. Comparison of 2 lung recruitment strategies in children with acute lung injury. Respir Care 2013;58(8):12801290.

15. Cruces P, Donoso A, Valenzuela J, Díaz F. Respiratory and hemodynamic effects of a stepwise lung recruitment maneuver in pediatric ARDS: a feasibility study. Pediatr Pulmonol 2013;48(11):1135-1143.

16. Boriosi JP, Sapru A, Hanson JH, Asselin J, Gildengorin G, Newman $\mathrm{V}$, et al. Efficacy and safety of lung recruitment in pediatric patients with acute lung injury. Pediatr Crit Care Med 2011;12(4):431-436.

17. Pediatric Acute Lung Injury Consensus Conference Group. Pediatric acute respiratory distress syndrome: consensus recommendations from the Pediatric Acute Lung Injury Consensus Conference. Pediatr Crit Care Med 2015;16(5):428-439. 


\section{Pediatric Mechanical Ventilation}

18. Jauncey-Cooke J, East CE, Bogossian F. Paediatric lung recruitment: a review of the clinical evidence. Paediatr Respir Rev 2015;16(2): 127-132.

19. Khemani RG, Sward K, Morris A, Dean JM, Newth CJ, NICHD Collaborative Pediatric Critical Care Research Network (CPCCRN). Variability in usual care mechanical ventilation for pediatric acute lung injury: the potential benefit of a lung protective computer protocol. Intensive Care Med 2011;37(11):1840-1848.

20. Smallwood CD, Walsh BK, Arnold JH, Gouldstone A. Equilibration time required for respiratory system compliance and oxygenation response following changes in positive end-expiratory pressure in mechanically ventilated children. Crit Care Med 2018; 46(5):e375-e379.

21. Whittenberger JL, McGregor M, Berglund E, Borst HG. Influence of state of inflation of the lung on pulmonary vascular resistance. J Appl Physiol 1960;15(Can't get issue from record):878-882.

22. Székely A, Sápi E, Király L, Szatmári A, Dinya E. Intraoperative and postoperative risk factors for prolonged mechanical ventilation after pediatric cardiac surgery. Paediatr Anaesth 2006;16(11): 1166-1175.

23. Kallio M, Peltoniemi O, Anttila E, Pokka T, Kontiokari T. Neurally adjusted ventilatory assist (NAVA) in pediatric intensive care-a randomized controlled trial. Pediatr Pulmonol 2015;50(1): 55-62.

24. Crulli B, Khebir M, Toledano B, Vobecky S, Poirier N, Emeriaud G. Neurally adjusted ventilatory assist after pediatric cardiac surgery: clinical experience and impact on ventilation pressures. Respir Care 2018;63(2):208-214.

25. Amirnovin R, Aghamohammadi S, Riley C, Woo MS, Del Castillo $\mathrm{S}$. Analysis of a pediatric home mechanical ventilator population. Respir Care 2018;63(5):558-564.
26. Bilharz JR, Wheeler CR, Walsh BK, Smallwood CD. A comparative analysis of ideal body weight methods for pediatric mechanical ventilation. Respir Care 2018;63(9):1079-1084

27. Ward SL, Quinn CM, Steurer MA, Liu KD, Flori HR, Matthay MA. Variability in pediatric ideal body weight calculation: implications for lung-protective mechanical ventilation strategies in pediatric acute respiratory distress syndrome. Pediatr Crit Care Med 2018;19(12): e643-e652.

28. (CDC) CfDCaP. Pediatric Ventilator-Associated Event (PedVAE). Available at: https://www.cdc.gov/nhsn/acute-care-hospital/pedvae/ index.html. Accessed November 12, 2018.

29. Cocoros NM, Kleinman K, Priebe GP, Gray JE, Logan LK, Larsen G, et al.; Pediatric Ventilator-Associated Conditions Study Team. Ventilator-associated events in neonates and children-a new paradigm. Crit Care Med 2016;44(1):14-22.

30. Chomton M, Brossier D, Sauthier M, Vallières E, Dubois J, Emeriaud G, Jouvet P. Ventilator-associated pneumonia and events in pediatric intensive care: a single center study. Pediatr Crit Care Med 2018;19(12):1106-1113.

31. Kirakli C, Ediboglu O, Naz I, Cimen P, Tatar D. Effectiveness and safety of a protocolized mechanical ventilation and weaning strategy of COPD patients by respiratory therapists. J Thorac Dis 2014;6(9): 1180-1186.

32. Kollef MH. Evaluating the value of the respiratory therapist: where is the evidence? Focus on the Barnes-Jewish Hospital experience. Respir Care 2017;62(12):1602-1610.

33. Krawiec C, Carl D, Stetter C, Kong L, Ceneviva GD, Thomas NJ. Challenges with implementation of a respiratory therapist-driven protocol of spontaneous breathing trials in the pediatric ICU. Respir Care 2017;62(10):1233-1240. 